\title{
Modeling of Covalent Bonding in Solids by Inversion of Cohesive Energy Curves
}

\section{Citation}

Bazant, Martin Z., and Efthimios Kaxiras. 1996. "Modeling of Covalent Bonding in Solids by Inversion of Cohesive Energy Curves." Physical Review Letters 77 (21): 4370-73. https:// doi.org/10.1103/physrevlett.77.4370.

\section{Permanent link}

http://nrs.harvard.edu/urn-3:HUL.InstRepos:41384109

\section{Terms of Use}

This article was downloaded from Harvard University's DASH repository, and is made available under the terms and conditions applicable to Other Posted Material, as set forth at http:// nrs.harvard.edu/urn-3:HUL.InstRepos:dash.current.terms-of-use\#LAA

\section{Share Your Story}

The Harvard community has made this article openly available.

Please share how this access benefits you. Submit a story.

Accessibility 


\title{
Modeling of Covalent Bonding in Solids by Inversion of Cohesive Energy Curves
}

\author{
Martin Z. Bazant and Efthimios Kaxiras \\ Department of Physics, Harvard University, Cambridge, MA 02138
}

(received 24 April 1996)

\begin{abstract}
We provide a systematic test of empirical theories of covalent bonding in solids using an exact procedure to invert $a b$ initio cohesive energy curves. By considering multiple structures of the same material, it is possible for the first time to test competing angular functions, expose inconsistencies in the basic assumption of a cluster expansion, and extract general features of covalent bonding. We test our methods on silicon, and provide the direct evidence that the Tersofftype bond order formalism correctly describes coordination dependence. For bond-bending forces, we obtain skewed angular functions that favor small angles, unlike existing models. As a proof-of-principle demonstration, we derive a $\mathrm{Si}$ interatomic potential which exhibits comparable accuracy to existing models.

PACS numbers: 61.50.Lt,34.20.Cf,33.15.Dj
\end{abstract}

Large-scale atomistic simulations are becoming increasingly important in the study of complex physical phenomena such as fracture, plastic deformation, twoand three-dimensional melting, epitaxial growth, shock wave propagation, friction, sintering, etc. Ideally, one would like to represent the atomic interactions in these simulations with a quantum mechanical approach, treating explicitly the electronic degrees of freedom. This is a computationally demanding proposition, tractable at present only for relatively small system sizes of order $10^{2}$ atoms. An alternative description is in terms of effective interatomic potentials which allow fast evaluation of energies and forces, making possible simulations involving more than $10^{8}$ atoms. The drawback in going from an explicit quantum treatment of electrons to an effective interatomic potential is a significant loss in accuracy that may undermine simulation results. In most cases, the microscopic mechanisms of greatest interest (for instance, bond formation or rupture) are precisely those which require high degree of transferability, that is, ability of the potential to describe accurately a wide range of local atomic environments.

Over a decade of experience has shown that such transferability is difficult to attain, especially in covalent solids, for inherently quantum effects such as bond bending and breaking, hybridization, charge transfer, and metalization. In the prototypical case of silicon, about thirty model potentials exist in the literature [1, including popular and innovative ones by Stillinger and Weber (SW) [2], Tersoff [3], and Chelikowsky et. al. [4]. Although the shortcomings of existing model potentials have been carefully documented, it has proven very difficult to improve them or to understand, even qualita- tively, the causes of their failures [1. [. [. Some theoretical arguments have been advanced to motivate the form of an effective interaction [6,7] and to derive potentials as approximations of quantum models \&8 11], but little specific theoretical guidance exists to aid in the development of new potentials. The most successful approach to date is to guess a functional form using physical intuition and then adjust parameters to fit a database of $a b$ initio structural energies [1]. The reliance on intuition and fitting leads to the two questions that motivate our work: (1) Is there an $a b$ initio justification for the functional form of an interatomic potential? and (2) Given a particular form, is there a systematic way to obtain new potentials directly from $a b$ initio energy data?

In this Letter, we present an exact procedure for inverting $a b$ initio energy data to obtain parameter-free many-body potentials [12]. The inversion approach was pioneered by Carlsson, Gelatt, and Ehrenreich (CGE) for the case of a pair potential [13], and since then the same formula has been applied with limited success by only a couple of authors [7, 14]. We revisit the inversion approach with the following innovations: (i) a recursive formulation that incorporates many-body interactions and strains other than uniform volume expansion; (ii) the requirement that $a b$ initio energies be exactly reproduced for relevant densities only (near the equilibrium solid and liquid densities); and (iii) the use of an overdetermined set of structures for the same material, which guarantees a wide range of relative atomic arrangements. These ideas form a general framework for analyzing functional forms and deriving potentials, as illustrated by application to Si. In this manner, we provide satisfactory answers to both questions posed in the previous paragraph. We analyze the two defining features of covalent bonding in two steps, 1. Pair bonding and 2. Angular forces.

1. Pair bonding: We begin with the simplest case of a pair potential, in which the cohesive energy $E[\phi]$ of an arbitrary structure is given by,

$$
E(r)=\sum_{i \neq j} \phi\left(R_{i j}\right)=\sum_{p=1}^{\infty} n_{p} \phi\left(s_{p} r\right),
$$

with atomic separations grouped into shells $S_{p}$ of radius $s_{p} r$ containing $n_{p}$ atoms each. Dilation of the lattice is achieved by varying the parameter $r$ with the structural quantities $\left\{s_{p}\right\}$ and $\left\{n_{p}\right\}$ fixed. Shells are numbered so that $s_{1}<s_{2}<s_{3}<\ldots$, and distances scaled so that $s_{1}=1$. A simple rearrangement of the terms in Eq. (11) yields the desired inversion formula for $\phi[E]$, 


$$
\phi(r)=\frac{1}{n_{1}}\left(E(r)-\sum_{p=2}^{\infty} n_{p} \phi\left(s_{p} r\right)\right) .
$$

Although the unknown potential appears on both sides of this equation, recursive substitution generates the explicit formula,

$$
\phi(r)=\frac{1}{n_{1}} E(r)-\sum_{p=2}^{\infty} \frac{n_{p}}{n_{1}^{2}} E\left(s_{p} r\right)+\sum_{p, q=2}^{\infty} \frac{n_{p} n_{q}}{n_{1}^{3}} E\left(s_{p} s_{q} r\right)-\ldots,
$$

which was originally derived by CGE invoking the linearity of the functional $E[\phi][13]$. Our recursive formulation generalizes to nonlinear functionals and suggests a simple computational procedure: If the tail of $\phi(r)$ is assumed known for $r>a$, then the potential is uniquely determined by solving the recursion in order of decreasing $r$ starting at $r=a$ (because $s_{p}>1$ for $p \geq 2$ ). An important case is that of finite range, i.e. $\phi(r)=0$ for $r \geq a$, as is typically assumed for interatomic potentials.

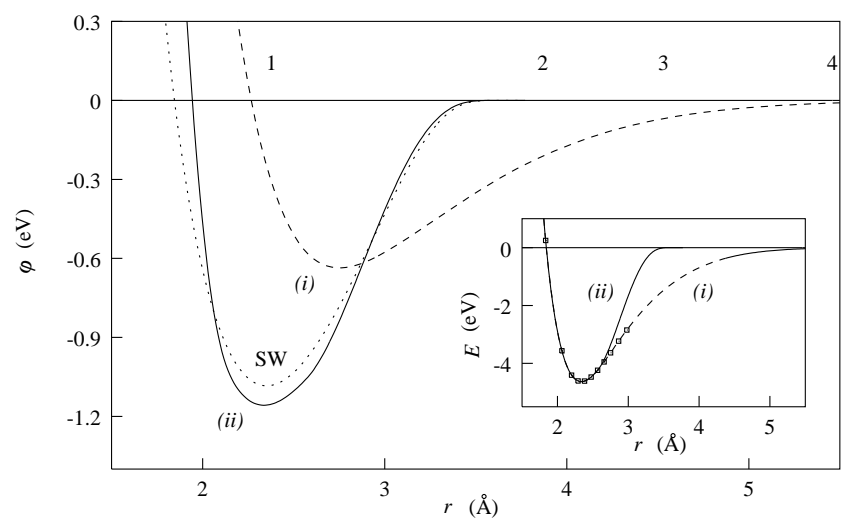

FIG. 1. The inverted pair potential for silicon (i) before and (ii) after a cutoff is imposed, compared with $\phi_{S W}(r)$ (dashed line). Numbers inside the figure indicate shell radii in the diamond lattice. The inset shows the diamond LDA data and the interpolant (i) before and (ii) after imposing a cutoff.

To illustrate the inversion procedure, we apply it to an $a b$ initio database consisting of cohesive energy curves for $\mathrm{Si}$ from density functional calculations in the local density approximation (LDA) 15]. In order to keep the procedure simple while still capturing the important local bonding characteristics, the database includes the following crystals: (i) the low-energy and low-coordination structures, diamond (Si-I), $\beta$-tin (Si-II) [16], BC-8 (SiIII) [17], and BCT-5 [18]; (ii) SC and FCC crystals for metallic behavior; and (iii) the graphitic structure for non-tetrahedral hybridization [19]. These structures have coordinations $4,6,4,5,6,12$, and 3 , respectively. We consider only atomic volumes smaller than $(3.54 \AA)^{3}$ to avoid the difficulty of LDA to represent accurately the energies of isolated atoms [20]. Smooth interpolation of the LDA data and extrapolation to infinite volume with an exponential tail are used. The LDA data points for the diamond lattice with the interpolant are shown in the inset of Fig. 1.

The inverted pair potential for the diamond curve, shown in Fig. 1, is clearly unphysical: Its long range and strong repulsion at the first neighbor distance contradict our intuitive understanding of covalent bonding. Similar , results have been obtained in previous work applying the CGE formula to metals [7,13] and semiconductors [14]. Our recursive approach reveals that these problems are inherent to the inversion process, which, in spite of being exact, stretches the assumption of a volume-independent potential to an unphysical extreme. Because inversion amounts to solving in order of decreasing distance from infinite separation, the tail of the potential comes from unscreened interactions between atoms in a low density gaseous phase [21]. The same tail is then used to describe long range interactions in a bulk crystal, which are presumably screened by the presence of closer atoms. While the nature of screening and its description by an effective potential are subjects of active research, it is obvious that distant atoms in a bulk crystal cannot interact in the same way as atoms with the same separation in a gas.

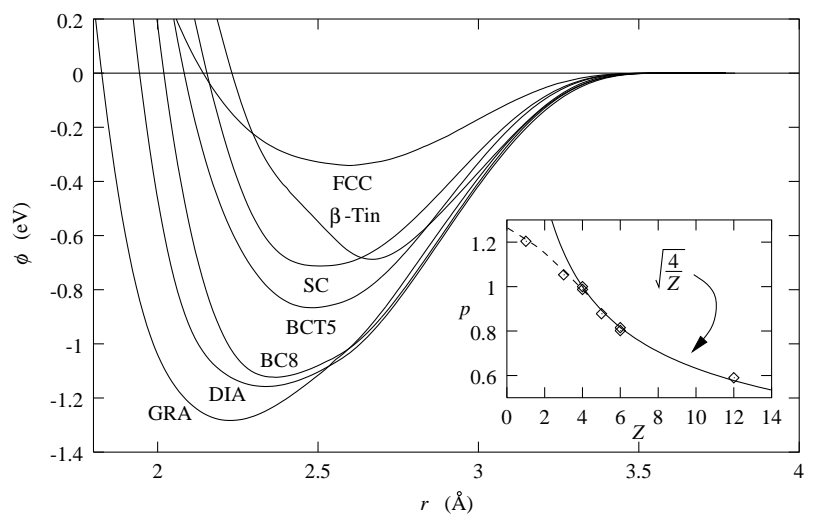

FIG. 2. Inverted pair potentials (with cutoff) for seven silicon bulk phases. The inset shows the implied bond order $p$ extracted from these curves (points) compared to $\sqrt{4 / Z}$ (line). $p(1)$ reflects the $\mathrm{Si}_{2}$ bond length and energy 11 .

To rectify the inversion procedure, we forgo the requirement that the potential exactly reproduce the entire cohesive energy curve. Instead, we focus on condensed volumes typical of solid and liquid environments, whose exact energies can be preserved with any choice of tail for the potential. For $\mathrm{Si}$, we find that exponential decay to a cutoff near the second-neighbor distance in the diamond lattice, $3.84 \AA$, generates potentials in good agreement with bonding theory. For example, as shown in Fig. 1, we force the energy curve to be zero for $r \geq a_{S W}=3.77118 \AA$ without disturbing energies within $10 \%$ of the equilibrium bond length, where covalent bonds are well-defined, to produce an inverted pair potential with a deep mini- 
mum at the first neighbor distance.

Applying the same procedure to the other curves in our database, we obtain the potentials of Fig. 2. The large discrepancy between them is direct evidence for a well-known fact: the energetics of silicon cannot be described by a pair potential alone [7]. Our results suggest, however, that an environment-dependent pair potential can describe the ideal bulk phases reasonably well. There is a clear coordination dependence to the curves: bond lengths (positions of the minima) increase, and bond strengths (depths of the minima) decrease with increasing coordination. This behavior can be described by the bond-order formalism, which is justified on grounds of theoretical arguments 6 9] as well as experience with empirical potentials [3,22,23]. In its simplest form, a bond order potential is given by,

$$
\phi(r, Z)=\phi_{R}(r)+p(Z) \phi_{A}(r),
$$

where $\phi_{R}$ and $\phi_{A}$ are monotonic repulsive and attractive terms, respectively, and $p(Z)$ gives the bond strength as a function of the coordination $Z$. The leading order approximation of the bond order is $p(Z) \propto Z^{-1 / 2}$. Since this comes from describing the local density of states by the bandwidth only, we expect the approximation to work well for the metallic phases with $Z>4$ (BCT-5, $\beta$ tin, SC, and FCC). For the covalent phases with $Z \leq 4$ (diamond, BC-8, and graphite), band shape effects become important and we expect significant departure from the $Z^{-1 / 2}$ behavior.

If the repulsive interaction $\phi_{R}$ were known, the bondorder term could be extracted directly from the $a b$ initio data, using $p(Z)=V_{A}\left(r_{0}\right) / V_{A}^{\text {dia }}\left(r_{0}\right)$, where $V_{A}=\phi-\phi_{R}$, $r_{0}$ is the minimum of the inverted potential $\phi$, and we set $p=1$ for the diamond lattice $(Z=4)$. The repulsive term, intended to represent an effective force between electrons due to Pauli exclusion, is the weakest link in bond-order models, since its form must be assumed and then fit to empirical data without theoretical guidance. Although the general trend is insensitive to the choice of $\phi_{R}$, we find that using $\phi_{R}=2 \phi_{R}^{S W}$, where $\phi_{R}^{S W}$ is the repulsive part of the SW potential, produces a $p(Z)$ which lies remarkably close to its expected behavior (see inset of Fig. 2).

2. Angular forces: While the energetics of bulk phases can be fairly well described by a bond order pair functional, it is well-known that many-body interactions with explicit angular dependence are required for silicon, for example, to stabilize the diamond lattice against shear strain [3,7]. As the simplest case of a many-body potential, we consider one with volume-independent pair terms and separable three-body terms like the potentials of SW and Kaxiras and Pandey [5]. The many-body energy, $F(r)=E(r)-V_{2}(r)$, formed by subtracting the pair terms $V_{2}(r)$ from the total energy $E(r)$, is expressed as a sum over pairs of bonds,

$$
F(r)=\sum_{i} \sum_{j \neq i} \sum_{k \neq i, k>j} g\left(R_{i j}\right) g\left(R_{j k}\right) h\left(\theta_{i j k}\right),
$$

where $\cos \theta_{i j k}=\hat{R}_{i j} \cdot \hat{R}_{j k}$. Following theory [6,7] and practice [1], we assume $F \geq 0$, which implies that the pair potential come from the diamond lattice inversion described above. A particular form for the angular term $h(\theta)$ must be assumed in order to invert $F[g, h]$ for the radial function $g[F, h]$. The procedure is the same as in the pair potential case: solve Eq. (5) for $g(r)$ to obtain a recursion. Grouping bonds into shells as above and taking the positive root of the resulting quadratic equation yields the desired expression,

$$
g(r)=\frac{-\beta(r)+\sqrt{\beta(r)^{2}+4 \alpha_{11}(F(r)-\gamma(r))}}{2 \alpha_{11}},
$$

where

$\alpha_{p q}=\sum_{r_{i j} \in S_{p}} \sum_{r_{i k} \in S_{q}} h\left(\theta_{i j k}\right), \quad \beta(r)=\sum_{p=2}^{\infty} \alpha_{1 p} g\left(s_{p} r\right)$, and $\gamma(r)=\sum_{p=2}^{\infty} \sum_{q=p}^{\infty} \alpha_{p q} g\left(s_{p} r\right) g\left(s_{q} r\right)$. In the $\alpha_{p p}$ sums, only $k>j$ contributes to avoid double counting. An explicit formula like the CGE pair potential can be obtained by recursive substitution with Eq. (6) and involves a tree of nested square roots. It is simpler to follow the same computational procedure as before, solving the recursion in order of decreasing distance starting at the cutoff. In principle, the same general procedure can be applied to determine radial functions of other forms or for higher order terms in a cluster expansion of the effective potential. For example, for a nonseparable three-body term involving three bond lengths, like the potential of Pearson et al. [24], the recursion comes from solving a cubic equation, and for a four-body term, a quartic equation.

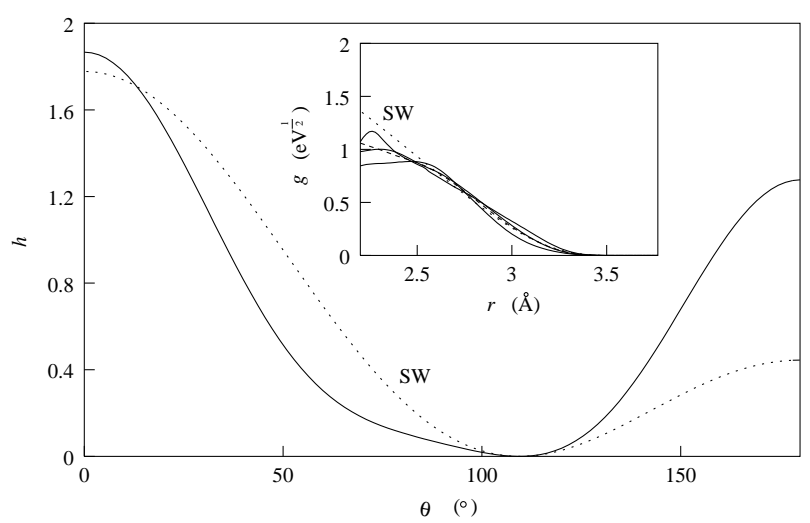

FIG. 3. An inverted angular function for silicon from the BC-8, BCT-5, and $\beta$-tin energy curves compared with $h_{S W}(\theta)$. The inset shows the collapse of the inverted $g(r)$ with the average curve (dashed line) and $g_{S W}(r)$ (dotted line).

As in the pair potential case, it is useful to invert more than one cohesive energy curve of the same material for $g[F, h](r)$. If the assumed angular dependence $h(\theta)$ (and two-body terms) were truly transferable, then 
the same radial function $g(r)$ would result from every inversion. Conversely, the greater the variance between the inverted $g(r)$, the less transferable is the assumed $h(\theta)$. This principle gives us a quantitative means of assessing the quality of angular functions directly from the $a b$ initio data. For example, although the SW angular function $h(\theta)=\left(\cos (\theta)+\frac{1}{3}\right)^{2}$, produces mediocre collapse of the inverted $g(r)$, it is fortuitously far better than taking $h(\theta)=\left(\cos (\theta)+\frac{1}{3}\right)^{4}$, due to the latter's flatness near the tetrahedral minimum.

Using the same principle, we can extract an optimal angular dependence from the $a b$ initio data by assuming a series expansion, $h(\theta)=\sum_{i=0}^{2} c_{i}\left(\cos (\theta)+\frac{1}{3}\right)^{2+i}$. For the curve shown in Fig. 3 (defined by $c_{0}=1, c_{1}=$ $-1.86, c_{2}=1.42$ ), the collapse of radial functions from the low energy phases is rather good, as seen in the inset of Fig. 3. A novel feature of the inverted angular function is its skew about the minimum to favor smaller angles. This is consistent with the conclusion that existing potentials tend to overpenalize angles smaller than $\pi / 2$ [1], which presumably leads to poor descriptions of surfaces, clusters, and certain defects. The skewed angular function also raises the energy of overcoordinated metallic structures relative to covalent ones by penalizing large angles. While it is typical to characterize metallic structures by the presence of small angles [4], we note that metallic structures tend to have angles near $\pi$ also. Covalent bonds are actually characterized by angles in the intermediate range, $\pi / 2$ to $2 \pi / 3$.

Although we are not attempting here to provide an improved potential for $\mathrm{Si}$, we have performed some tests of the potential obtained by the inversion just described [25] (in this proof-of-principle demonstration, we omit coordination dependence for practical reasons). We find that it performs as well as the popular SW and Tersoff potentials without fitting to any defect structures, for energies of other silicon bulk phases, defects such as interstitials and vacancies, generalized stacking faults, the concerted exchange diffusion mechanism [5], and (100) and (111) surface reconstructions. Considering the database employed in the inversion, we conclude that many important features of chemical bonding are contained in cohesive energy curves for ideal bulk phases.

In conclusion, we have presented a general procedure for inverting cohesive energy curves to obtain many-body effective interatomic potentials. By inverting ab initio cohesive energy curves for silicon, we have demonstrated how general features of bonding are revealed. Elsewhere we will describe extensions of these ideas, for example, to the inversion of energy curves for shear strains to obtain the angular function $h[F, g](\theta)$ directly. The inversion procedure provides a systematic method for deriving interatomic potentials and a unique tool for understanding their general limitations through the direct use of $a b$ initio data. It is hoped that this tool will lead to potentials with improved transferability, a goal that has proven elu- sive when pursued by intuitive arguments and fitting of databases.

MZB acknowledges a Computational Science Graduate Fellowship from the Office of Scientific Computing of the Department of Energy. This work was supported in part by ONR grant \#N00014-93-I-0190.

[1] H. Balamane, T. Halicioglu, and W. A. Tiller, Phys. Rev. B 46, 2250 (1992) and references therein.

[2] F. Stillinger and T. Weber, Phys. Rev. B 31, 5262 (1985).

[3] J. Tersoff, Phys. Rev. Lett. 56, 632 (1986); Phys. Rev. B 37, 6991 (1988); 38, 9902 (1988);

[4] J. R. Chelikowsky, J. C. Phillips, M. Kamal, and M. Strauss, Phys. Rev. Lett. 62, 292 (1989).

[5] E. Kaxiras and K. Pandey, Phys. Rev. B 38, 736 (1988).

[6] A. E. Carlsson and N. Ashcroft, Phys. Rev. B 27, 2101 (1983); A. E. Carlsson, Phys. Rev. B 32, 4866 (1985).

[7] A. E. Carlsson, in Solid State Physics: Advances in Research and Applications, edited by H. Ehrenreich and D. Turnbull (Academic, New York, 1990), 43, 1.

[8] G. C. Abell, Phys. Rev. B 31, 6184 (1985).

[9] D. G. Pettifor, Springer Proc. in Physics 48, 64 (1990).

[10] A. E. Carlsson, P. A. Fedders and C. W. Myles, Phys. Rev. B 41, 1247 (1990).

[11] J. S. McCarly and S. T. Pantelides, Bull. Am. Phys. Soc. 41, 264 (1996).

[12] M. Z. Bazant and E. Kaxiras, in Materials Theory, Simulations and Parallel Algorithms, ed. by E. Kaxiras, J. Joannopoulos, P. Vashista, and R. Kalia, MRS Proceedings 48 (Materials Research Society, Pittsburgh, 1996).

[13] A. Carlsson, C. Gelatt, and H. Ehrenreich, Phil. Mag. A 41 (1980).

[14] J. Wang, K. Zhang, and X. Xie, J. Phys. C 6, 989 (1994).

[15] We use a plane wave basis with a 12 Ry cutoff and 512 points in the full Brillouin zone for reciprocal space integrations. These parameters guarantee sufficient accuracy.

[16] M. Yin and M. Cohen, Phys. Rev. B 26, 5668 (1982).

[17] M. T. Yin, Phys. Rev. B 30, 1773 (1984).

[18] M. Mehl and L. Boyer, Phys. Rev. B 43, 9498 (1991); E. Kaxiras and L. Boyer, Phys. Rev. B 50, 1535 (1994).

[19] To capture the planar nature of graphitic bonds, energies are computed for planar expansion with fixed $c$.

[20] N. Moll, M. Bockstedte, M. Fuchs, E. Pehlke, and M. Scheffler, Phys. Rev. B 52, 2550 (1995).

[21] Our assertion is supported by Fig. 4 of Ref. 7 in which the tail of the inverted potential for $\mathrm{Cu}$ is seen to overlap with $\mathrm{Cu}_{2}$ binding energy curve.

[22] B. W. Dodson, Phys. Rev. B 35, 2795 (1987);

[23] B. Bolding and H. Anderson, Phys. Rev. B 41, 10568 (1990).

[24] E. M. Pearson, T. Takai, T. Halicioglu, and W. A. Tiller, J. Crystal Growth 70, 33 (1984).

[25] The potential consists of the diamond $\phi(r)$ with cutoff (Fig. 1(ii)), the optimized angular function (Fig. 3), and the averaged $g(r)$ (dashed curve from the inset of Fig. 3). 